\title{
Phenotypic Characterization and Genetic Mapping of An Open-hull Sterile Mutant in Rice
}

\author{
Yoye Yu, Rihua Piao, Wenzhu Jiang, Sunghan Kim, and Hee-Jong Koh* \\ Department of Plant Science, Plant Genomics and Breeding Institute, and Research Institute of Agriculture and Life Sciences, \\ Seoul National University, Seoul 151-921, Korea
}

\begin{abstract}
Rice hulls remain closed throughout the ripening period to maintain internal humidity of the grains. An Open-hull sterile mutant was induced by N-methyl-N-nitrosourea (MNU) treatment on Sinsunchalbyeo rice, a japonica type. This mutant showed open hulls even in the ripening stages and fully mature grains. In addition, several altered characteristics were observed, including of narrowed palea, decreased grain size, partial pollen sterility and erect panicle. Microscopic analysis showed that the palea was positioned slightly inside the lemma, and the size of palea decreased in the mutant. Genetic analysis of $F_{2}$ and $F_{3}$ segregation populations derived from the cross between the Open-hull sterile mutant (Oryza sativa ssp. japonica) and Milyang23 (O. sativa ssp. indica) indicated that the Open-hull trait was controlled by a single recessive allele. The fine-mapping with STS (sequence tagged site) markers revealed that the mutant gene was located on the short arm of chromosome 3 . We were able to narrow it down until $30.6 \mathrm{~Kb}$ where three candidate genes were found.
\end{abstract}

Keywords Rice, Open-hull sterile, Mutant, Mapping

\section{INTRODUCTION}

In angiosperms, flower is the most important organ for reproduction and seed dispersal, and grasses including the three major cereals, rice, wheat, and maize, produce grains from their inflorescences. As with other members of the grass family, rice inflorescence is organized in spikelets, each consisting of two sterile lemmas, two rudimentary glumes, and a floret which has one carpel, six stamens, two lodicules, and lemma and one palea (Kyozuka et al. 2000; Bommert et al. 2005; Kater et al. 2006). The glumes, lemma and palea are modified leaves that enclose the reproductive parts of the flower and found only in the grass. Rice seed is protected with hull, a hard-cover structure composed largely of lemma and palea. In general, about two-third of the total surface of a rice seed is covered with the lemma due to its larger size than the palea, in which two edges of lemma enclose the edges of palea from outside, so that they do not separate easily (Hoshikawa 1989).
Several mutants which led to abnormal development of lemma and palea have been reported in the past ten years. The leafy hull sterile 1 mutant $(l h s 1)$, the locus of which was mapped to the chromosome 3 (Jeon et al. 2000), typically exhibited phenotypic alteration of elongated leafy palea and lemmas that features an open hull. The palealess (pal) mutant flower has all wild-type floral organs except the palea that is replaced by two leaf-like organs and was mapped to the short arm of chromosome 6 (Luo et al. 2005). The supernumerary bract (snb) mutant is characterized with the empty glumes and lodicules transformed into lemma/ palea-like organs (Lee et al. 2006). The rep1, a mutation in the gene regulating palea identity and development, RETARDED PALEA1 (REP1), displayed significantly retarded palea development (Yuan et al. 2009). The stunted lemma palea 1 (slp1), though controlled by a single gene (Wang et al. 2011), showed extreme degeneration of both the lemma and palea.

In crop breeding, hybrid that has been created by crossing

Received Mar 5, 2013; Revised Mar 18, 2013; Accepted Mar 19, 2013; Published March 31, 2013

*Corresponding author Hee-Jong Koh, heejkoh@snu.ac.kr, Tel: +82-2-880-4541, Fax: +82-2-873-2056 
two different parental strains had many benefits (Crow 1948). Such crosses generally result in an $F_{1}$ heterosis that showing superior agronomic qualities over their parents in higher yield, stronger resistance to diseases, more efficient use of soil nutrients, and better weed control (Birchler 2003). Rice hybrids have shown $15-20 \%$ higher yield potential than inbred rice varieties under field conditions (Virmani 1982), and have also exhibited superior ability to perform better under drought and salinity (Akbar and Yabuno 1975). Generation of $F_{1}$ hybrids in rice has been limited by the self-pollination character of the crop, and thus commercial rice hybrids have been mainly developed by using a male sterility system (Yu 1997), in which pollens are made unviable genetically or non-genetically so that rice spikelets become incapable of setting seeds through selfing.

Here, we report a spikelet mutant in rice, named Open-hull sterile mutant, which produces abnormal hull and an open flower. Throughout phenotypic characterization, genetic analysis, and molecular mapping, this study aims to identify the candidate genes responsible for the Open-hull sterile mutant phenotype, thereby to provide a better prospect in development of male sterility lines for the hybrid rice program.

\section{MATERIALS AND METHODS}

\section{Plant materials}

An Open-hull sterile mutant was obtained by N-methylN-nitrosourea (MNU) treatment on Sinsunchalbyeo cultivar, a Japonica type. Seeds of the Open-hull sterile mutant were taken from an $\mathrm{M}_{12}$ line, 77296-3, the pedigree name of which was Sinsunchalbyeo M-B-17-1-2-1-1-1-2-1-2. For the genetic analysis and fine-mapping of such mutant, the $F_{2}$ population and a segregating $F_{3}$ lines were used. The $F_{2}$ population was generated from self-pollination of $F_{1}$ plant obtained by the cross between the Open-hull sterile mutant line mentioned above and Milyang 23 (O. sativa ssp. indica). Both $F_{2}$ and $F_{3}$ populations were grown at the Experimental Farm of Seoul National University in Suwon using conventional cultural practices during the 2010 and 2011 rice-growing season, respectively. Plant materials were seeded on the plastic tunnel seedbed and thirty-day-old seedlings were transplanted to irrigated field condition at one seedling per hill with a $30 \times 15 \mathrm{~cm}$ of spacing between seedlings.

\section{Pollen fertility observation}

Pollen fertility was determined using the $\mathrm{I}_{2}$-KI staining method (Wang et al. 2006). Pre-flowering spikelets were gathered and fixed in FAA solution (ethanol $50 \%$, acetic acid $5 \%$, formaldehyde $3.7 \%$ ). Six anthers were sampled randomly and then removed from the spikelets. Pollens were placed on slides with $1 \% \mathrm{I}_{2}$-KI solution, and nipped into pieces using forceps to make the pollen grains spill out. The pollen fertility was examined under optical microscope according to the morphology and staining gradation. Pollen grains that were round in shape and stained black were considered to be viable.

\section{Histochemical Analyses}

Paraffin-embedded sections of spikelet samples were prepared according to Li et al. (2009) method with slight modifications. Spikelets were collected at pre-flowering stage and fixed in FAA solution (ethanol $50 \%$, acetic acid $5 \%$, formaldehyde $3.7 \%$ ) and stored at $4{ }^{\circ} \mathrm{C}$ for 1 day. The fixed spikelets were dehydrated by soaking for 2 hours each in a gradient ethanol series $(70 \%, 85 \%, 95 \%)$ and then incubated in $100 \%$ ethanol overnight. Spikelet samples were cleared by soaking for 2 hours in the clearing solution series consisting of $75 \%$ ethanol $/ 25 \%$ histo-clear, $50 \%$ ethanol $/ 50 \%$ histo-clear, $25 \%$ ethanol $/ 75 \%$ histo-clear, followed by soaking in $100 \%$ histo-clear solution overnight. For paraffin infiltration, the spikelet samples were soaked for 2 hours in the solution series of $75 \%$ histo-clear $/ 25 \%$ paraffin, $50 \%$ histo-clear $/ 50 \%$ paraffin, $25 \%$ histo-clear $/ 75 \%$ paraffin, and $100 \%$ paraffin at $55^{\circ} \mathrm{C}$ overnight. The paraffin infiltrated samples were embedded in paraffin blocks and then cut by microtome into $12 \mu \mathrm{m}$ sections. The sections were transferred onto gelatin (sodium salicylate $1 \mathrm{~g}$, egg white $50 \mathrm{ml}$, Glycerol $50 \mathrm{ml}$ ) -coated glass slides and dried at $42^{\circ} \mathrm{C}$ for 1 day. The sections were deparaffinized with $100 \%$ xylen for $10 \mathrm{~min}$ followed by hydration by soaking for 2 min each in $50 \%$ ethanol $/ 50 \%$ xylen, $100 \%$ ethanol and sterile water. After staining in $1 \%$ safranin $/ 30 \%$ ethanol for $30 \mathrm{~s}$ and washed with sterile water twice, the samples 
were soaked for $2 \mathrm{~min}$ each in 30\% ethanol, 50\% ethanol, $70 \%$ ethanol, $85 \%$ ethanol, $95 \%$ ethanol. Finally, the sections were cleared by soaking twice in $100 \%$ xylen and mounted in Canada balsam. The cross sections of spikelets were observed with an optical microcopy at 100X magnification.

\section{Genetic mapping}

A total of $1530 \mathrm{~F}_{2}$ plants derived from a cross between Open-hull sterile mutant and Milyang23 were used. Total genomic DNA were extracted from young leaves according to the cetyltrimethyl-ammonium bromide (CTAB) method as described by Causse et al. (1994) with slight modification. Equal amounts of DNA from each of the 10 individual plants were pooled into a single bulked sample for bulked segregant analysis (BSA) strategy as described by Michelmore et al. (1991). After following the Open-hull sterile mutant phenotype using BSA strategy, fine mapping was conducted with STS markers developed based on the sequence differences between the indica and japonica rice (Cho et al. 2007) which were available in rice genome sequence data (http://www. ncbi.nlm.nih.gov;http://www. gramene.org; http://www.rgp.dna.affrc.go.jp), plus additional STS markers (Table 1) designed in silico using Primer3 software version 0.4.0 (http://frodo.wi.mit.edu/primer3).

\section{RESULTS}

Characterization of Open-hull sterile mutant in rice

The main characteristic of the Open-hull sterile mutant is abnormal spikelets that result in open flowers. In contrast to wild-type spikelets that are enclosed within the palea and lemma, the mutants had an Open-hull because their spikelets had a gap between the palea and lemma (Fig.1A). While the phenotypes of spikelets in the Open-hull sterile mutants varied greatly, the most typical shape of the mutant spikelet featured open hull, but having the palea and lemma developed normally. On the other hand, some of the mutant spikelets had a slightly narrowed palea and were half open. Another common type of the mutant spikelet showed abnormal palea development that rendered the seed uncovered with the hull completely (Fig.1B).

Other than the panicle shape, the overall appearance of the Open-hull sterile mutant plants showed no visible abnormalities including in plant height and the leaf shape, as compared with the wild-type plants (Fig. 1C). As grains fill, the panicle of the wild-type droops to bend in a plane curve, whereas the panicle of the Open-hull sterile looks like an erect panicle even after maturation (Fig.1D).

To determine when the spikelet of the mutant starts to open, we observed the wild type and the Open-hull sterile mutant plants at various developmental stages. While the

Table 1. The PCR-based molecular markers used in $F_{2}$ genetic mapping of the Open-hull sterile mutant.

\begin{tabular}{cccc}
\hline \hline STS marker & Forward primer $\left(5^{\prime}-3^{\prime}\right)$ & Reverse primer $\left(3^{\prime}-5\right.$ ') & $\begin{array}{c}\text { Product } \\
\text { size }(\mathrm{bp})\end{array}$ \\
\hline 146619 & TTTACAAGAGCGCATACATCTG & TGCAACCTGAGTGGAGTTAATG & 193 \\
$146619-2$ & ATTTTCTCTGCACCCTGTTCAC & CTTTCCCAATGGAATATCAGGT & 168 \\
$146619-3$ & TGTGTTCCCCTGTCTACTTTCA & GAATCCACTCCCATGTATTCGT & 182 \\
105732 & GATAAAATGTCTCAGCGCCACT & GAGACAGGAATCGAACTCTTGC & 198 \\
$105732-2$ & TCTGAGAAAATAGTTAGGAAATGATGA & CAAGGTCCCTAACACCAAGAAA & 197 \\
134241 & ACATGAGTCACGAAACGATGAA & GGGTGAGATGTTTTGCTTTGTT & 200 \\
$134241-2$ & GCCCTTTCCTCCTTTAAGAAAC & CAGGAGAGGATAAATTGGGAGA & 181 \\
135158 & TCTGTTCATGTGTCTCCGATCT & CATGATCCTATGTTCGGTCGAT & 171 \\
104474 & CTTCGCGTGGAGCAGTACTAT & ACACACGAACAATCTCAAATGC & 198 \\
107226 & TCATACAATTCGTTGCGTTTTC & AAGTTCGTCTCGCAGTTACAGG & 190 \\
$107226-2$ & AACAAACCCTAGCTACGAACCA & GACCCACTAGCTCGGATAAATG & 158 \\
104473 & AACCAATTAATCATGCCCTAATG & ACTTCCAAAACCAGTTTCATCG & 163 \\
\hline
\end{tabular}



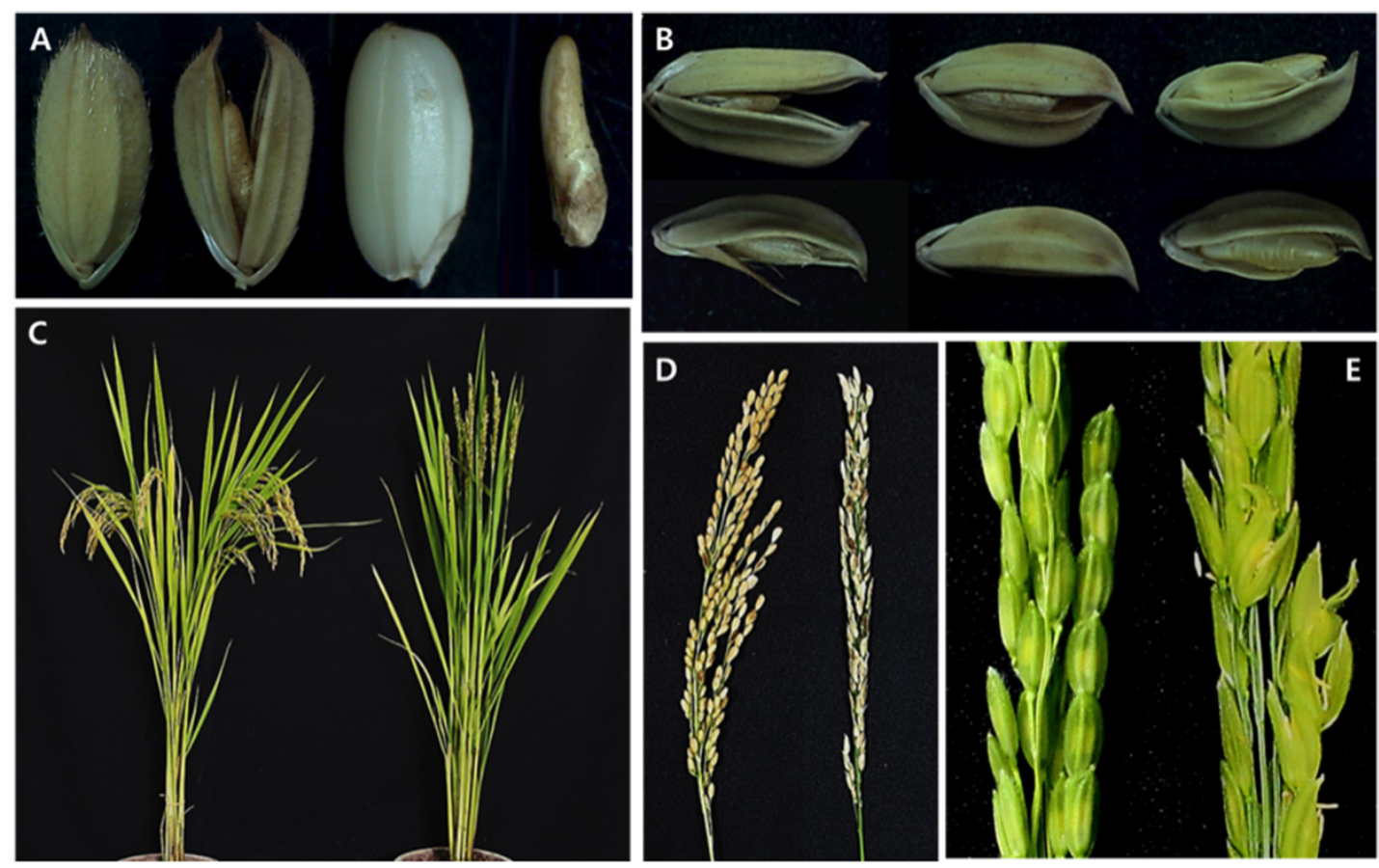

Fig. 1. Overall phenotypes of wild type and Open-hull sterile mutant.

A. Spikelets and seeds phenotypes of the wild type (left) and the Open-hull sterile mutant (right).

B. Various spikelets phenotype of the Open-hull sterile mutant.

C. Plant of wild type (left) and Open-hull sterile mutant (right).

D. Panicle shape of wild type (left) and Open-hull sterile mutant (right). E. Spikelet of wild type (left) and Open-hull sterile mutant (right) after at the ripening stage.

spikelet of the wild-type opened at flowering time and then closed right after, the spikelet of Open-hull sterile mutant started opening from the beginning of the heading stage, and remained open after flowering time (Fig. 1E).

Agronomic traits such as grain shape and fertility rate were also compared. The grain width and thickness were significantly decreased in the Open-hull sterile mutant compared to those in the wild type (Fig. 1A). Consistent with this, the grain weight, as determined by the weight of 100 brown rice grain, also decreased by about $30 \%$ in the Open-hull sterile mutant $(1.41 \mathrm{~g}$ in the mutant vs. $2.01 \mathrm{~g}$ in the wild type: Table 2).

\section{Anatomical structure of the Open-hull sterile mutant}

The phenotype of the Open-hull sterile mutant at anatomical level was also examined by preparing paraffin-

Table 2. Descriptive statistics of agronomic traits of the Open-hull sterile mutant and wild-type.

\begin{tabular}{cccccccc}
\hline \hline Traits & $\mathrm{HD}^{\mathrm{z})}$ & $\begin{array}{c}\mathrm{GL} \\
(\mathrm{mm})\end{array}$ & $\begin{array}{c}\mathrm{GW} \\
(\mathrm{mm})\end{array}$ & $\begin{array}{c}\mathrm{GT} \\
(\mathrm{mm})\end{array}$ & $\mathrm{GS}$ & $\begin{array}{c}\text { Brown rice 100-grain } \\
\text { weight }(\mathrm{g})\end{array}$ & Fertility rate (\%) \\
\hline Wild-type & $8 / 14$ & 5.54 & 2.89 & 2.16 & 1.92 & 2.01 & 83.1 \\
Mutant & $8 / 15$ & 5.40 & 1.99 & 1.37 & 2.78 & 1.41 & 19.8 \\
Difference & $\mathrm{ns}^{\mathrm{y})}$ & $*$ & $* *$ & $* *$ & $* *$ & $* *$ & $* *$ \\
\hline
\end{tabular}

${ }^{\mathrm{z})} \mathrm{HD}$, is a the abbreviation of heading date; GL, for grain length; GW, for grain width; GT, for grain thickness; GS, for grain shape (length/width ratio).

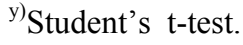

*, $0.01<P<0.05 ; * *, p<0.01$; NS, reffered to not significantly different at the 0.05 level of significance. 

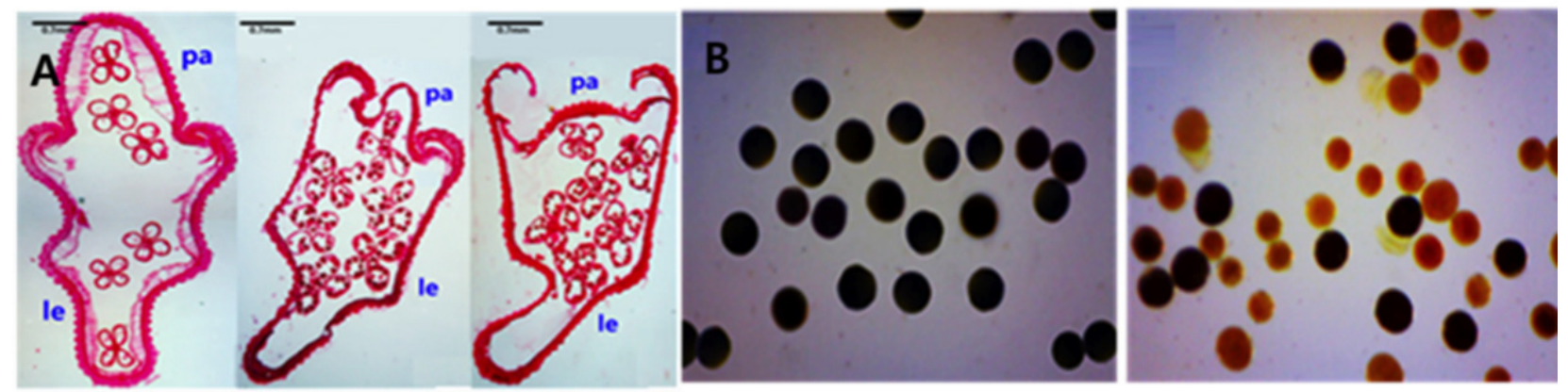

Fig. 2. A. Spikelet cross sections of the wild-type (left) and Open-hull sterile mutants (middle and right. pa;palea, le; lemma. B. I2-KI pollen fertility test of the wild type (left) and Open-hull sterile mutant (right). Pollen grains stained dark black and light yellow were considered to be fertile and sterile, respectively.

embedded spikelet sections which revealed degenerated palea that was significantly reduced in size and positioned slightly inside the lemma (Fig. 2A). In some cases, the palea of the mutant was positioned completely inside the lemma, with no interlock between the palea and lemma (Fig. 2A).

\section{Pollen fertility test.}

In order to obtain a better insight into the reason for the spike sterility in the Open-hull sterile mutant, pollens of the mutant and wild type were subjected to fertility test by staining them with $1 \% \mathrm{I}_{2}-\mathrm{KI}$ solution. Pollens of the wildtype spikelets were fully fertile, showing the characteristic round and filled appearance and were stained dark with $\mathrm{I}_{2}$-KI solution. By contrast, the Open-hull sterile mutant pollens showed partial pollen sterility with irregular shape and size of the pollens that are only lightly stained appearing yellowish in color, or unstained at all (Fig. 2B), suggesting that the partial sterility of the mutant pollen is largely responsible for the decreased seed-setting rates observed in the mutant, thus conferring sterility.

\section{Genetic analysis of the Open-hull sterile mutant}

When the Open-hull sterile mutants were crossed to wild type Milyang 23 (Tongil type), all $\mathrm{F}_{1}$ progeny individuals appeared normal indicating that the mutant phenotype is controlled by recessive allele(s). $F_{2}$ seeds were further generated for the mapping population and the segregating phenotypes of normal and Open-hull types in the population were scored. Of the total 1530 individual plants of $F_{2}$ population, 1250 plants showed the normal type and 280 plants showed the Open-hull type, which did not fit the 3:1 ratio of the Mendelian inheritance. This result was likely due to the segregation distortion which often occurs in the $\mathrm{F}_{2}$ population derived from the inter-subspecific (indical japonica) cross, where partial hybrid sterility was important factor on distorted segregation (Lin et al. 1992). To clarify the segregation ratio, we harvested seeds from a population of heterozygous $F_{2}$ plants and grewow the $F_{3}$ plants. In support of this, The normal type plants were 483 and the Open-hull type plants were 163 in the $F_{3}$ population, restoring the expected Mendelian segregation of 3:1 ratio. All of these results suggest that the Open-hull trait was controlled by a single recessive allele (Table 3 ).

Table 3. Segregation of the normal and the Open-hull sterile mutant types in $F_{1}, F_{2}$ and $F_{3}$ populations. $X^{2} 0.05(1)=3.841$

\begin{tabular}{|c|c|c|c|c|c|c|}
\hline \multirow{2}{*}{ Cross combination } & \multirow{2}{*}{ Generation } & \multicolumn{2}{|c|}{ No. of plant } & \multirow{2}{*}{ Total } & \multirow{2}{*}{$\begin{array}{c}X^{2} \\
(3: 1)\end{array}$} & \multirow{2}{*}{$p$} \\
\hline & & Wild-type & Mutant & & & \\
\hline \multirow{3}{*}{$\begin{array}{c}\text { Mutant / } \\
\text { Milyang } 23\end{array}$} & $\mathrm{~F}_{1}$ & 35 & 0 & 35 & - & - \\
\hline & $\mathrm{F}_{2}$ & 1,250 & 280 & 1,530 & 36.267 & $>0.05$ \\
\hline & $\mathrm{F}_{3}$ & 483 & 163 & 646 & 0.019 & $<0.01$ \\
\hline
\end{tabular}



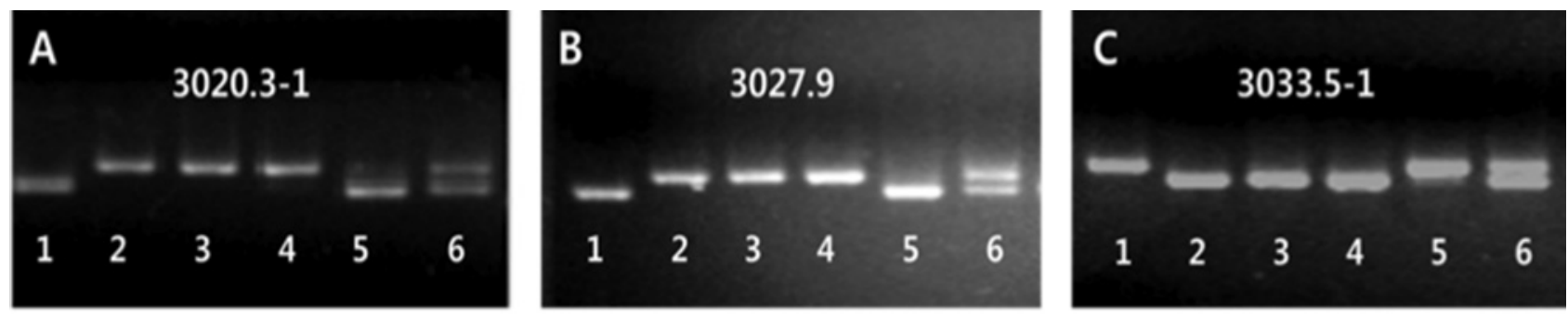

Fig. 3. Bulked segregant analysis (BSA) of wild-type and mutant bulks from $F_{2}$ population.

A C. STS (sequence tagged site) markers linked to the Open-hull sterile mutant locus. 1: Milyang 23 (Tongil-type rice). 2: Sinsunchallbyeo(Japonica type) Wild-type. 3, 4: Mutant bulks. 5, 6: Wild-type bulks.

\section{Genetic mapping of Open-hull sterile mutant}

The genetic mapping of the Open-hull sterile mutant was conducted by using 1250 individual plants of $F_{2}$ population derived from the cross between the mutant and Milyang 23. To identify markers linked to the mutant gene, bulked segregation analysis (BSA) was performed with 72 STS markers distributed over 12 rice chromosomes (Michelmore et al. 1991). Three STS markers, including 3020.3-1, 3027.9 and 3033.5-1, which are located on the short arm of chromosome 3 , showed significant polymorphisms between the bulked DNA samples of the Open-hull sterile mutant and wild-type plants (Fig. 3A-C). Using these three polymorphic markers, the linkage analysis was conducted by genotyping the $F_{2}$ population. As a result, locus of the gene for the Open-hull sterile mutation was mapped to a region flanked by the two markers 3027.9 and 3033.5-1, which spans 5.6 cM. Using 12 additional markers, this locus was then subsequently narrowed down to a region of $191 \mathrm{~Kb}$ in length.

To further refine the position of the gene, a total of 646 individual plants of $\mathrm{F}_{3}$ population obtained by selfing of the
$F_{2}$ plants were screened with 9 additional STS markers (Table 4). Consequently, the Open-hull sterile mutant gene was identified within $30.6 \mathrm{~kb}$ region between two flanking markers, 105732-3 and 105732-8, which were present in two overlapping BAC clones (AP135158 and AP105732). Based on available sequence annotation databases (http:// www.ncbi.nlm.nih.gov; http://www.tigr.org http://www. gramene.org), three candidate genes existed in this region; including LOC_Os03g11614.1, which encodes OsMADS1, LOC_Os03g11630.1, a locus for a putative MuDR transposon, and LOC_Os03g11640.1, (Fig. 4), which predicts a short open reading frame (237 bp) of unknown function. Among these, OSMADS1 appears to be the most likely candidate responsible for the Open-hull sterile mutant phenotype, since it has been identified as a key regulator of floral meristem identity and the one linked to the leafy hull sterile1 (lhs1) mutant phenotype (Jeon et al. 2000). That prompted us to examine the sequence of OSMADS1 locus region of the Open-hull sterile mutant including the promoter and open reading frame sequences, but no

Table 4. The PCR-based molecular markers used in $F_{3}$ genetic mapping of the Open-hull sterile mutant.

\begin{tabular}{cccc}
\hline \hline STS marker & Forward primer $\left(5^{\prime}-3^{\prime}\right)$ & Reverse primer $\left(3^{\prime}-5{ }^{\prime}\right)$ & Product size $(\mathrm{bp})$ \\
\hline $105732-3$ & GCATGGAGTATGTACCCCCTAT & CTTGAACACATGACATATACTTTGC & 155 \\
$105732-5$ & TATCGAAATGCTGCCGAAAT & GCAGCTCTGTGTCCAGTTCA & 137 \\
$105732-6$ & GGTCAAAGTGTTATCAACCCAGA & GCGCATATATACCCCCATCA & 116 \\
$105732-7$ & ATGTGGGACCACCTCGTAGT & TCCAATGGCTGCTTTATTCc & 139 \\
$105732-8$ & AGAACATCAACTGGGCTTTCA & GCACACAAGATAAACCCAATCA & 136 \\
$105732-9$ & TTTCTTGTCGTTTGGGCTTC & CGCACAAGAGAAACAGGTGA & 145 \\
$105732-10$ & TTTACCCTCGGCGATAGAGA & GTCGCCATCACGATGAACT & 124 \\
$105732-11$ & CCAGCAAAAACGTAACCTTGA & TGCAGCTAGAGAATGGTCGAT & 140 \\
\hline
\end{tabular}


A

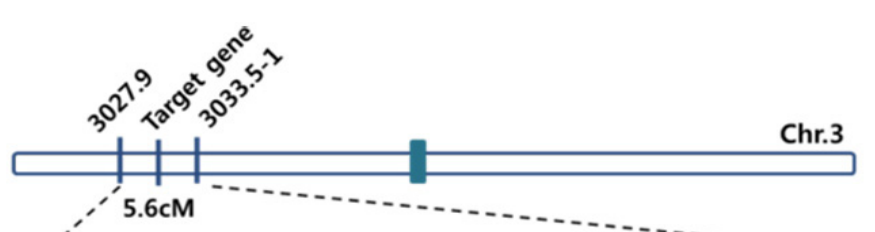

B

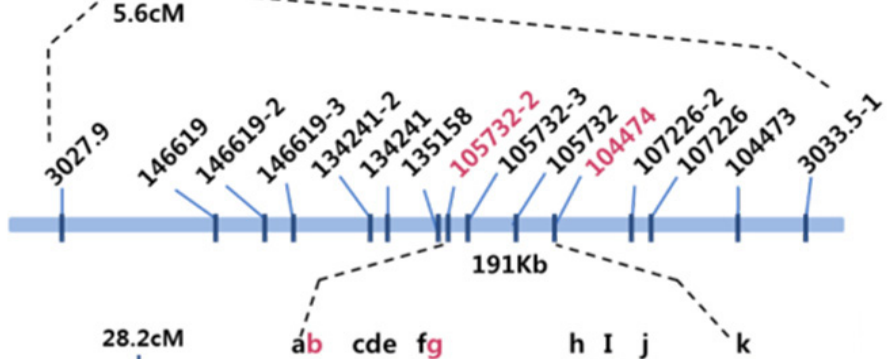

C

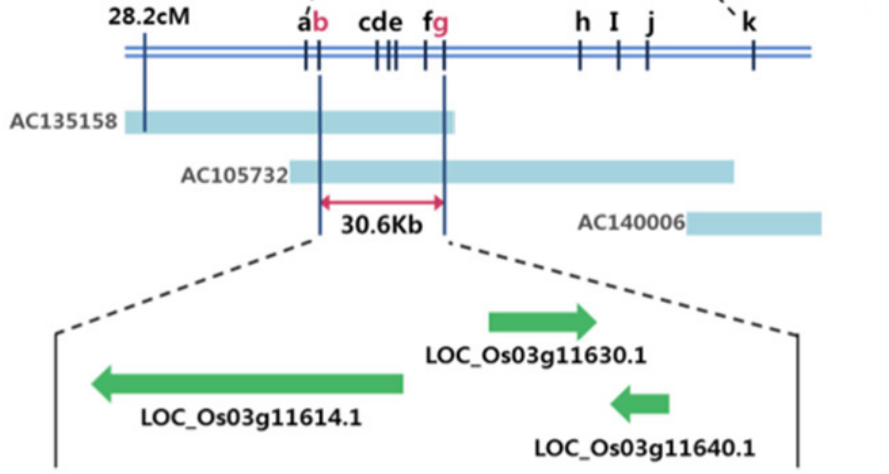

Fig. 4. Genetic and physical maps of the Open-hull sterile mutant.

A. A Schematic genetic linkage map of Open-hull sterile mutant gene. The mutant gene was primarily mapped on the short arm of chromosome 3, based on the bulked segregant analysis (BSA) of $F_{2}$ individuals. The genetic distances (centi Morgan $[\mathrm{cM}]$ ) between flanking markers are indicated. B. Physical map of the mutant gene, which was made by genotyping of $1530 \mathrm{~F}_{2}$ individuals using STS markers. The physical distance between adjacent markers, 105732-3 and 104474 (pink letters), was $191 \mathrm{~Kb}$. C.The mutant gene was fine mapped to an interval of $30.6 \mathrm{~Kb}$ by genotyping of $646 \mathrm{~F}_{3}$ individuals which derived from $\mathrm{F}_{2}$ plants using additional STS markers (b-j). BAC contigs covering the mutant gene which published by IRGSP are shown. a, 105732-2; b, 105732-3; c, 105732-4; d, 105732-5; e, 105732-6; f, 105732-7; g, 105732-8; h, 105732-9; i, 105732-10; j, 105732-11; k, 104474. (D) Three putative genes, which were annotated in RAP-DB database, LOC_Os03g11614.1, LOC_Os03g11630.1 and LOC_Os03g11640.1, existed in this 30.6Kb region.

sequence alterations have been found in the region. Further sequencing is in progress.

\section{DISCUSSION}

In this study, we identified and characterized a rice mutant showing an open flower. A number of mutant flowers have been described in rice, such as multiple pistil-1 (mp-1), multiple pistil-2 (mp-2), and the floral-organnumber mutant (fon(t)), in which aberrations in not only the floral organ shape but also the flower organ numbers were observed in common (Librojo and Khush 1986, Nagasawa et al. 1996; Li Y et al. 2007). On the contrary, the Open-hull sterile mutant displayed only an alteration of spikelet form. In wild-type rice, flowers are enclosed strongly by outer whorl organs, lemma and palea, and this probably makes the plant propagate largely by self- pollination. As the the hull of Open-hull sterile mutant was open since the beginning of the heading stage, its propensity for cross pollination is expected to be higher than the wild type rice, which could potentially be a useful agronomic trait. However, being unable to close at the seed maturing time rendering the seeds unprotected from the external environment during a critical period of the seed development (Fig. 1A), the grain size and weight were significantly decreased in the mutant compared to the wild type (Table 2). Unlike some flowering mutant of rice, such as slp1 
(stunted lemma palea 1) that showed dwarfism in plant height and short, fragile leaves in addition to the defective floral morphology (Wang et al. 2011), no alterations in the vegetative phenotypes including increase or reduction in plant height and the leaf shape were observed in the Openhull sterile mutant, other than the panicle shape (Fig. 1C).

The result of our mapping data, which narrowed down the location of the gene responsible for the Open- hull sterile phenotype within a $30.6 \mathrm{~kb}$ region of chromosome 6 , where three genes including OSMADS1 are present. Although it is quite possible that a sequence alteration in the OSMADS1 is directly responsible for the phenotype of Open-hull sterile mutant plant, considering its reported role in floral organ development (Jeon et al. 2000), no sequence changes were found in the genomic region defining the OsMADSl, thus ruling out the possibility of the OSMADSI being the locus of Open-hull sterile allele at least at present. This conclusion is also supported by the fact that the actual phenotype of Open-hull sterile mutant, although similar, can be distinguishable from that of the leafy hull sterile1 (lhsl) mutant phenotype caused by OSMADS1 mutation as reported by Jeon et al. (2000).

The mutant plant had only about $19 \%$ fertility, which was in sharp contrast with the average seed setting rate of $83 \%$ seen in the wild type plants (Table.2). This lightweighted spikelet of the mutant plants is thought to be attributable to the erect phenotype of the mutant panicle, which does not bend or droop upon maturation, unlike those of wild type plants (Fig. 1D). Through pollen fertility test, it was revealed that the very low seed setting rate observed in the Open-hull sterile mutant was mainly due to the partial sterility of the mutant pollens. This was further confirmed by reciprocal crosses between the mutant and the wild type, where emasculated mutant flowers pollinated by wild type pollens produced normal, fertile seeds implying that anthers in the mutant flower function normal. Based on this finding, it may be possible to utilize the Open-hull sterile mutant as a convenient male sterile line for generating $\mathrm{F}_{1}$ hybrids.

With the genetic analysis and fine mapping of the Openhull sterile mutant to the short arm of chromosome 3 described in present study, a framework for expanding the knowledge of rice hull development and associated the mutant traits could be established, and the map based cloning of the Open-hull sterile mutant gene, which is to be followed as a next stepm can provide more insight into understanding the mutant gene function and molecular mechanism for its interaction with other floral genes.

\section{ACKNOWLEDGEMENTS}

This work was supported by a grant from the NextGeneration BioGreen 21 Program (Plant Molecular Breeding Center, No. PJ008125), Rural Development Administration, Republic of Korea.

\section{REFERENCE}

Akbar M and Yabuno, T. 1975. Breeding for saline resistant varieties of rice. III. Response of $F_{1}$ hybrids to salinity in reciprocal crosses between Jhona 349 and Magnolia. Japan. J. Breed. 25:215-220.

Bommert P, Satoh N, Jackson D and Hirano HY. 2005. Genetics and evolution of inflorescence and flower development in grasses. Plant Cell Physiol. 46:69-78.

Causse MA, Fulton TM, Cho YG, Ahn SN, Chunwongse J, Wu K, Xiao J, Yu Z, Ronald PC, Harrington SE and et al. 1994. Saturated molecular map of the rice genome based on an interspecific backcross population. Genet. 138:1251-1274.

Cho YI, Jiang W, Chin JH, Piao Z, Cho YG, McCouch S and Koh HJ. 2007. Identification of QTLs associated with physiological nitrogen use efficiency in rice. Mol. Cells 23:72-79.

Jeon JS, Jang S, Lee S, Nam J, Kim C, Lee SH, Chung YY, Kim SR, Lee YH, Cho YG and An G. 2000. Leafy hull sterile1 is a homeotic mutation in a rice MADS box gene affecting rice flower development. Plant Cell 12:871-884.

Kater MM, Dreni L and Colombo L. 2006. Functional conservation of MADS-box factors controlling floral organ identity in rice and Arabidopsis. J. Exp. Bot. 57:3433-3444.

Kyozuka J, Kobayashi T, Morita M and Shimamoto K. 2000. Spatially and temporally regulated expression of rice MADS box genes with similarity to Arabidopsis class A, B and C genes. Plant Cell Physiol. 41:710-718. 
Lee DY, Lee JW, Moon SO, Park SY and An GH. 2006. The rice heterochronic gene SUPERNU-MERARY BRACT regulates the transition from spikelet meristem to floral meristem. Plant J. 49:64-78.

Li H, Xue D, Gao Z, Yan M, Xu W, Xing Z, Huang D, Qian Q and Xue Y. 2009. A putative lipase gene EXTRA GLUME1 regulates both empty-glume fate and spikelet development in rice. Plant J. 57:593-605.

Li Y, Xu PZ, Zhang HY, Peng H, Zhang QF, Wang XD and Wu XJ. 2007. Characterization and identification of a novel mutant fon(t) on floral organ number and floral organ identity in rice. J. Genetics and Genomics 34:730-737.

Librojo AL and Khush GS. 1986. Chromosomal location of some mutant genes through the use of primary trisomics in rice. In Rice Genetics, Proceeding of the International Rice Genetics Symposium, Mania: Island Press, Inc., pp.249-255.

Lin SY, Ikehashi H, Yanagihara S and Kawashima A. 1992. Segregation distortion via male gametes in hybrids between indica and japonica or wild-compatibility varieties of rice (Oryza sativa L). Theor. Appl. Genet. 84:812-818.

Luo Q, Zhou K, Zhao X, Zeng Q, Xia H, Zhai W, Xu J, Wu X, Yang $H$ and Zhu L. 2005. Identification and fine mapping of a mutant gene for palealess spikelet in rice. Planta. 221:222-230.

Michelmore RW, Paran I and Kesseli RV. 1991. Identification of markers linked to disease-resistance genes by bulked segregant analysis: a rapid method to detect markers in specific genomic regions by using segregating populations. PNAS 88:9828-9832.

Nagasawa N, Miyoshi M, Sano Y, Satoh H, Hirano H, Sakai H and Nagato Y. 2003. SUPERWOMAN1 and DROOPING $L E A F$ genes control floral organ identity in rice. Development 130:705-718.

Yu SB, Li JX, Xu CG, Tan YF, Gao YJ, Zhang Q and Saghai Maroof MA. 1997. Importance of epistasis as the genetic basis of heterosis in an elite rice hybrid. PNAS 94: 9226-9231.

Yuan Z, Gao S, Xue DW, Luo D, Li LT, Ding SY, Yao X, Wilson Zoe A, Qian Q and Zhang DB. 2009. RETARDED PALEA1 controls palea development and floral zygomorphy in rice. Plant Physiol. 149:235-244.

Wang SS, Wang CS, Tseng TH, Hou YL and Chen KY. 2011. High-resolution genetic mapping and candidate gene identification of the SLP1 locus that controls glume development in rice. Theor. Appl Genet. 122(8):1489-1496.

Wang, SS, Wang CS, Tseng TH, Hou YL and Chen KY 2011. High-resolution genetic mapping and candidate gene identification of the SLP1 locus that controls glume development in rice. Theor. Appl. Genet. 122:1489-1496.

Wang Z, Zou Y, Li X, Zhang Q, Chen L, Wu H, Su D, Chen Y, Guo J, Luo D, Long Y, Zhong Y and Liu YG 2006. Cytoplasmic male sterility of rice with boro II cytoplasm is caused by a cytotoxic peptide and is restored by two related PPR motif genes via distinct modes of mRNA silencing. Plant Cell. 18:676-687. 